\title{
THE $\beta$-CHANGE BY FINSLER METRIC OF C-REDUCIBLE FINSLER SPACES IN FINSLER GEOMETRY
}

\section{Khageswar Mandal}

\begin{abstract}
This paper considered about the $\beta$-Change of Finsler metric $L$ given by $L^{*}=f(L, \beta)$, where $f$ is any positively homogeneous function of degree one in $\mathrm{L}$ and $\beta$ andobtained the $\beta$-Change by Finsler metric of $\mathrm{C}$-reducible Finsler spaces. Also further obtained the condition that a C-reducible Finsler space is transformed to a C-reducible Finsler space by a $\beta$-change of Finsler metric.
\end{abstract}

Keywords: $\beta$-change, Finsler Metric, C-reducible Finsler Spaces.

\section{INTRODUCTION}

\section{Finsler space}

Suppose that we are given a function $\mathrm{L}\left(\mathrm{x}^{\mathrm{i}}, \mathrm{y}^{\mathrm{i}}\right)$ of the line element $\left(\mathrm{x}^{\mathrm{i}}, \mathrm{y}^{\mathrm{i}}\right)$ of a curve defined in $\mathrm{R}$. We shall assume $\mathrm{L}$ as a function of class at least $\mathrm{C}_{5}$ in all its $2 \mathrm{n}$ arguments. If we define the infinitesimal distance $\mathrm{ds}$ between two point $\mathrm{p}\left(\mathrm{x}^{\mathrm{i}}\right)$ and $\mathrm{Q}\left(\mathrm{x}^{\mathrm{i}}+\mathrm{dx}\right)$ of $\mathrm{R}$ by the relation

$$
\mathrm{ds}=\mathrm{L}\left(\mathrm{x}^{\mathrm{i}}, \mathrm{y}^{\mathrm{i}}\right)
$$

then the manifold $\mathrm{m}^{\mathrm{n}}$ equipped with the fundamental function $\mathrm{L}$ defining the metric (1) is called a Finsler space (Cartan, 1934; Asanor, 1985; Rund, 1959; Matsumoto, 1992; Matsumoto et al., 1976; Finsler, 1918; Pandey et al. 1997; Kropina, 1961), if $\mathrm{L}\left(\mathrm{x}^{\mathrm{i}}, \mathrm{dx}^{\mathrm{i}}\right)$ satisfies the following conditions.

Condition $\mathrm{A}$ : The function $\mathrm{L}\left(\mathrm{x}^{\mathrm{i}}, \mathrm{y}^{\mathrm{i}}\right)$ is positively homogeneous of degree one in yi.e.

$$
\mathrm{L}\left(\mathrm{x}^{\mathrm{i}}, \mathrm{ky}\right)=\mathrm{KL}\left(\mathrm{x}^{\mathrm{i}}, \mathrm{y}^{\mathrm{i}}\right), \mathrm{k}>0
$$

1 Dr. Mandal is Associate Professor, Mathematics Department, Padma Kanya Multiple Campus, Kathmandu, TU. 
Condition B: The function $\mathrm{L}\left(\mathrm{x}^{\mathrm{i}}, \mathrm{y}^{\mathrm{i}}\right)$ is positive is not all $\mathrm{y}^{\mathrm{i}}$ vanish simultaneously i.e.

$$
\mathrm{L}\left(\mathrm{x}^{i}, \mathrm{y}^{i}\right)>0 \text { with }_{i}^{\Sigma}(y i)^{2} \neq 0
$$

Condition C: The quadratic form

$$
\dot{\partial}_{i} \dot{\partial}_{j} L^{2}(x, y) \xi^{i} \xi^{j}=\frac{\partial^{2} L^{2}(x, y)}{\partial y^{i} \partial y^{j}} \xi^{i} \xi^{j}
$$

is assmed to be positive definite for any variable $\xi^{i}$

\section{C-reducible Finsler space}

A Finsler space of dimension n, more than two, is called C-reducible if $\mathrm{C}_{\mathrm{ijk}}$ is written in the form (Prasad et al. 2013; Prasad et al. 1998; Asanor, 1981; Matsumoto, 1986; Matsumoto et al. 1980).

$$
C_{i j k}=\frac{1}{n+1}\left(h_{i j} C_{k}+h_{j k} C_{i}+h_{k i} C_{j}\right),
$$

Where $C_{i}=C_{i j k} g^{j k}$ is the torsion vector and $h_{i j}$ is the angular metric tensor given by $h_{i j}=g_{i j}-l_{i} l_{j}$.

\section{Matsumoto Change of Finsler metric}

Let $F^{n}=\left(M^{n}, L\right)$ be an n-dimensional Finsler space on a differentiable manifold $\mathrm{M}^{\mathrm{n}}$, equipped with the fundamental function $\mathrm{L}(\mathrm{x}, \mathrm{y})$.

(Shukla et. al. 2012) introduced the transformation of Finsler metric called Matsumoto change of Finslermetric given by

$$
\bar{L}(x, y)=\frac{L^{2}}{L-B}
$$

where $\beta(x, y)=b_{i}(x) y^{i}$ is a one form on $M^{n}$

\section{Exponential Change of Finsler metric}

Let $\mathrm{F}^{\mathrm{n}}=\left(\mathrm{M}^{\mathrm{n}}, \mathrm{L}\right)$ be an $\mathrm{n}$-dimensional finsler space on a differential manifold $\mathrm{M}^{\mathrm{n}}$, equipped with the fundamental function $\mathrm{L}(\mathrm{x}, \mathrm{y})$. (Prasad et al. 2013) introduced an exponential change of finsler metric given by

$$
\bar{L}(x, y)=L e^{\beta / L}
$$

where $\beta(x, y)=b i(x) y^{i}$ is a one form on $M^{n}$ 


\section{$\beta$-change of Finsler metric:}

Let $\mathrm{F}^{\mathrm{n}}=\left(\mathrm{M}^{\mathrm{n}} ; \mathrm{L}\right)$ be an $n$-dimensional Finsler space on the differentiable manifold $M^{n}$ equipped with the fundamental function $L(x, y)$, (Prasad et.al 2013; Synge, 1925; Shibata, 1984; Park et al. 2001) considered the change of Finsler metric given by

$$
L^{*}(x, y)=f(L, \beta),
$$

where $f$ is positively homogeneous function of degree one in $\mathrm{L}$ and $\beta$ and $\beta$ given by $\beta(x, y)=b_{i}(x) \mathrm{y}^{i}$ is a one-form on $\mathrm{M}^{\mathrm{n}}$. The Finsler space $\left(\mathrm{M}^{\mathrm{n}} ; \mathrm{L}^{*}\right)$ obtained from $F^{n}$ by the $\beta$-change (5) will be denoted by $F^{* n}$.

The Homogeneity of $f$ in (5) gives

$$
L f_{1}+\beta f_{2}=f
$$

where the subscripts ' 1 ' and ' 2 ' denote the partial derivatives with respect to $L$ and $\beta$ respectively.

Differentiating (6) with respect to $L$ and $\beta$ respectively, we get

$$
\mathrm{LF}_{11}+\beta \mathrm{f}_{12}=0 \text { and } \mathrm{Lf}_{12}+\beta \mathrm{f}_{22}=0
$$

Hence, we have

which gives

$$
\frac{f_{11}}{\beta^{2}}=-\frac{f_{12}}{L \beta}=\frac{f_{22}}{L^{2}}
$$

$$
f_{11}=\beta^{2} \mathrm{w}, f_{22}=L^{2} \mathrm{w}, f_{12}=-\beta L \mathrm{w}
$$

where Weierstrass function $w$ is positively homogeneous function of degree -3 in Land $\beta$. Therefore

$$
L \mathrm{w}_{1}+\beta \mathrm{w}_{2}+3 \mathrm{w}=0
$$

Again $w_{2}$ is positively homogeneous of degree -4 in Land $\beta$, so

$$
L \mathrm{w}_{21}+\beta \mathrm{w}_{22}+4 \mathrm{w}_{2}=0
$$

Throughout the paper we frequently use above equations (6) to (8) without quoting them.

The concept of concurrent vector field has been given by (Matsumoto et al. 1974; Tachibana, 1950), which is defined as follows: 
The vector field $b_{i}$ is said to be a concurrent vector field if
(i) $b_{i j}=-g_{i j}$
(ii) $\left.b_{i}\right|_{j}=0$

where small and long solidus denote the $h$-and $v$-covariant derivatives respectively.

It has been proved by Matsumoto that $b_{i}$ and its contravariant components $b^{i}$ are functions of coordinates alone. Therefore from (9) (ii), we have

$$
C_{i j k}^{b^{i}}=0
$$

\section{FUNDAMENTAL QUANTITIES OF F*n}

To find the relation between fundamental quantities of $F^{n}$ and $F^{* n}$, we use the following results:

$$
\dot{\partial}_{i} \beta=b_{i}, \quad \dot{\partial}_{i} L=L_{i}, \quad \dot{\partial}_{j} l_{i}=L^{-1} h_{i j}
$$

where $\dot{\partial}_{i}$ stands for $\frac{\partial}{\partial y^{i}}$ and $h_{i j}$ are components of angular metric tensor of $F^{n}$ given by $h_{i j}=g_{i j}-l_{i} l_{j}=L \dot{\partial}_{i} \dot{\partial}_{j} L$

The successive differentiation of (1) with respect to $y^{i}$ and $y^{j}$ gives:

$$
\begin{aligned}
& l_{i}^{*}=f_{1} l_{i}+f_{2} b_{i} \\
& h_{i j}^{*}=\frac{f f_{1}}{L} h_{i j}+f L^{2} w m_{i} m_{j},
\end{aligned}
$$

where $m_{i}=b_{i}-\frac{\beta}{L} l_{i}$

The quantities corresponding to $F^{*_{n}}$ will be denoted by putting star on the top of those quantities.

From (11) and (12) we get the following relations between metric tensors of $F^{n}$ and $F^{* n}$.

$$
\begin{aligned}
& g_{i j}^{*}=\frac{f f_{1}}{L} g_{i j}-\frac{p \beta}{L} l_{i} l_{j}+\left(f L^{2} w+f_{2}^{2}\right) b_{i} b_{j}+p\left(l_{i} b_{j}+l_{j} b_{i}\right), \\
& \text { where } p=\left(f_{1} f_{2}-f \beta L w\right) .
\end{aligned}
$$


The contravariant components of the metric tensor of $F^{*_{n}}$ will be derived from (13) as follows:

$$
\begin{aligned}
& g^{*_{i j}}=\frac{L}{f f_{1}} g^{i j}+\frac{p L^{3}}{f^{3} f_{1} t}\left(\frac{f \beta}{L^{2}}-\Delta f_{2}\right) l^{i} l^{j}-\frac{L^{4} w}{f f_{1} t} b^{i} b^{j}-\frac{p L^{2}}{f^{2} f_{1} t}\left(l^{i} b^{j}+l^{j} b^{i}\right) \\
& t=f_{1}+L^{3} w \Delta, \quad \Delta=b^{2}-\frac{\beta^{2}}{L^{2}}
\end{aligned}
$$

putting $q=3 f_{2} w+f_{2} w$, we find that
(a) $\dot{\partial}_{i} f=\frac{f}{l} l_{i}+f_{2} m_{i}$,
(b) $\quad \dot{\partial}_{i} f_{1}=-\beta L w m_{i}$,
(c) $\dot{\partial}_{i} f_{2}=L^{2} w m_{i}$,
(d) $\dot{\partial}_{i} w=-\frac{3 w}{L} l_{i}+w_{2} m_{i}$,
(e) $\dot{\partial}_{i} b^{2}=-2 C . . ._{i}$,
(f) $\quad \dot{\partial}_{i} \Delta=-2 C ._{._{i}}-\frac{2 \beta}{L^{2}} m_{i}$,

and

(a) $\dot{\partial}_{i} p=\beta \operatorname{Lqm}_{i}$,

(b) $\dot{\partial}_{i} t=-2 L^{3} w c .{ } t+\left(L^{3} \Delta w_{2}-3 \beta L w\right) m_{i}$,

(c) $\dot{\partial}_{i} q=-\frac{3 q}{L} l_{i}+\left(4 f_{2} w_{2}+3 w^{2} L^{2}+f w_{22}\right) m_{i}$,

where, '.' denotes the contraction with $b^{i}$, viz $C . . ._{i}=C_{j k i} b^{j} b^{k}$

Differentiating (13) with respect to $y^{k}$, using and (16), we get the following relation between the Cartan's $C-$ tensors $\left(C_{i j k}^{*}=\frac{1}{2} \dot{\partial}_{k} g_{i j}^{*}\right.$ and $\left.C_{i j k}=\frac{1}{2} \dot{\partial}_{k} g_{i j}\right)$ :

$$
C_{i j k}^{*}=\frac{f f_{1}}{L} C_{i j k}+\frac{p}{2 L}\left(h_{i j} m_{k}+h_{j k} m_{i}+h_{k i} m_{j}\right)+\frac{q L^{2}}{2} m_{i} m_{j} m_{k}
$$

It is to be noted that

$$
m_{i} l^{i}=0, m_{i} m^{i}=\Delta=m_{i} b^{i}, h_{i j} j^{j}=0, h_{i j} m^{j}=, h_{i j} b^{j}=m_{i}
$$


where $m^{i}=g^{i j} m_{j}=b^{i}-\frac{\beta}{L} l^{i}$

To find $C_{j k}^{* i}=g^{* i h} C_{h j k}^{*}$ we use (14), (18), (19), we get

$C_{j k}^{*_{i}}=C_{j k}^{i}+\frac{p}{2 f f_{1}}\left(h_{j k} m^{i}+h_{j}^{i} m_{k}+h_{k}^{i} m_{j}\right)+\frac{q L^{3}}{2 f f_{1}} m_{j} m_{k} m^{i}$

$-\frac{L}{f t} C_{j k} b n^{i}-\frac{p L \Delta}{2 f^{2} f_{1} t} h_{j k} n^{i}-\frac{2 p L+L^{4} \Delta q}{2 f^{2} f_{1} t} m_{j} m_{k} n^{i}$,

where $n^{i}=f L^{2} w b^{i}+p l^{i}$

We have the following relations corresponding to the vectors with components $n^{i}$ and $m^{i}$ :

$C_{\cdot i j k} m^{i}=C_{j k}, \quad C_{i j k} n^{i}=f L^{2} w c_{j k}, \quad m_{i} m^{i}=f L^{2} w \Delta$

\section{$\beta$-CHANGE OF C-REDUCIBLE FINSLER SPACE}

Let $F^{n}$ be a C-reducible Finsler space. Then (Matsumoto, 1972)

$C_{h j k}=\frac{1}{n+1}\left(h_{h j} C_{k}=h_{h k} C_{j}=h_{j k} C_{i}\right)$

where $C_{k}=C_{h j k} g^{h j}$

Using equation (22) in equation (18), we get

$C_{h j k}^{*}=\left(p_{k} h_{h j}+p_{j} h_{h k}+p_{h} h_{j k}\right)+\frac{q L^{2}}{2} m_{i} m_{j} m_{k}$

where $\quad p_{k}=\frac{f f_{1}}{L(n+1)} C_{k}+\frac{p}{2 L} m_{k}$

Using equation (12) in equation (23), we get

$C_{h j k}^{*}=\frac{L}{f f_{1}}\left(p_{k} h_{h j}^{*}+p_{j} h_{h k}^{*}+p_{h} h_{j k}^{*}\right)+q_{h} m_{j} m_{k}+q_{j} m_{h} m_{k}+q_{k} m_{j} m_{h}$

where $q_{h}=\frac{q L^{2}}{6} m_{h}-\frac{L^{3} w}{f_{1}} p_{h}$

Now suppose that the transformation (1) is such that $(n+1)\left(f_{1} w_{2}+3 \beta L w^{2}\right) m_{h}=6 f_{1} w C_{h}$ then $q_{h}=0$. So equation (25) reduces to

$C_{h j k}^{*}=\frac{L}{f f_{1}}\left(p_{k} h_{h j}^{*}+p_{j} h_{h k}^{*}+p_{h} h_{j k}^{*}\right)$ 


$$
\begin{aligned}
& \text { which will give } \frac{C_{k}^{*}}{n+1}=\frac{L}{f f_{1}} p_{k} \text {, so that } \\
& C_{n j k}^{*}=\frac{1}{n+1}\left(C_{k}^{*} h_{n j}^{*}+C_{j}^{*} h_{n k}^{*}+C_{n}^{*} h_{j k}^{*}\right)
\end{aligned}
$$

Hence $F^{n}$ is also a C-reducible. Therefore we have the following:

Theorem (1) Under the $\beta$-change of Finsler metric with the condition $(n+1)\left(f_{1} w_{2}+3 \beta L w^{2}\right) m_{n}=6 f_{1} w C_{n}$ the C-reducible Finsler space by $\beta$-change of Finslermeticis trans-formed to a $\mathrm{C}$-reducible Finsler space.

In the theorem (1) we have assumed that $(n+1)\left(f_{1} w_{2}+3 \beta L w^{2}\right) m_{n}=6 f_{1} w C_{n}$. However if this condition is not satisfied then a C-reducible Finsler space may not be transformed to a $\mathrm{C}$-reducible Finsler space. In the following we discuss under what condition a C-reducible Finsler space is transformed to a C-reducible Finsler space by $\beta$-change of Finsler metric.

In both the spaces $F^{n}$ and $F^{* n}$ are C-reducible then from (22) and its corresponding equation for $F^{* n}$ we find, on using (18), that

$$
\begin{aligned}
& \frac{f L^{2} w}{t}\left[\left(\mathrm{Q}_{h} m_{j} m_{k}+\mathrm{Q}_{j} m_{h} m_{k}+\mathrm{Q}_{k} m_{j} m_{h}\right)-f_{1}\left(\mathrm{C} . ._{h} h_{j k}+C . ._{j} h_{h k}+C . . \cdot k_{k} h_{j h}\right)\right] \\
& =\left(\frac{p}{2 L}-\frac{f f_{1} r}{L(n+1)}\right)\left(h_{j k} m_{h}+h_{h j} m_{k}+h_{h k} m_{j}\right)+\left(\frac{p L^{2}}{2}-3 f L^{2} w r\right) m_{h} m_{j} m_{k}
\end{aligned}
$$

where $Q_{h}=C_{h}-L^{3} w C . . ._{h}$ and $r=(n-2) p t+f_{1}\left(3 p+L^{3} q \Delta\right)$

Thus, we have the following:

Theorem (2) A C-reducible Finsler space is transformed to a C reducible Finsler space by a $\beta$-change of Finsler metric if and only if (29) holds.

The condition (29) of theorem (2) is too complicated to study any geometrical concept of Finsler space. So we consider that our $\beta$ in $\beta$-change of Finsler metric is such that $b_{i}$ is a concurrent vector field so that $\mathrm{C}_{\mathrm{i}_{\mathrm{i}}}=0$, $\mathrm{C} ._{\mathrm{i}}=0$. Hence equation (29) reduces to 


$$
\begin{aligned}
f L^{2} w\left(C_{n} m_{j} m_{k}\right. & \left.+C_{j} m_{n} m_{k}+C_{k} m_{j} m_{n}\right) \\
& =\left(\frac{p}{2 L}+\frac{f f_{1} r}{2 L}\right)\left(h_{j k} m_{n}+h_{n j} m_{k}+h_{n k} m_{j}\right) \\
& +\left(\frac{q L^{2}}{2}-3 f^{2} w r\right) m_{n} m_{j} m_{k}
\end{aligned}
$$

Contracting this equation with $g^{j k}$, we find

$$
2 f L^{3} w \Delta C_{n}=\left\{(n+1)\left(p-f f_{1} r\right)+\left(q L^{3}-6 f^{2} L w r\right) \Delta\right\} m_{n}
$$

Hence we have the following:

Theorem (3) If a C-reducible Finsler space is transformed to a $C$-reducibleFinsler space by a concurrent $\beta$-change of Finsler metric, then the vector $C_{h}$ is along the direction of the vector $m_{h}$.

\section{CONCLUSION}

a) The C-reducible finsler space is transformed to a $\mathrm{C}$-reductibleFinsler space under the $\beta$-change of Finsler metric if

(i) if $(\mathrm{n}+1)\left(\mathrm{f}_{1} \mathrm{w}_{2}+3 \beta \mathrm{Lw}^{2}\right) \mathrm{m}_{\mathrm{h}}=\mathrm{bf}_{1} \mathrm{wc}_{\mathrm{h}}$.

(ii) if and only if (29) holds

b) If a $\mathrm{C}$-reducible finsler space is transformed to a $\mathrm{C}$-reducible finsler space by a concurrent $\beta$-change of finsler metric, then the vector $c_{h}$ is along the direction of the vector $\mathrm{m}_{\mathrm{h}}$

\section{REFERENCES}

Asanor, G. S. (1981). C-reducible finsler spaces : Finsler spaces with randers metrics and kropina metrics. J. Sov. Math 17: 1610-1624.

Asanor, G. S. (1985). Finsler geometry, relativity and gange theories. Phy. D. Reidel Publ. Co.Dordrecht, Hotland.

Cartan, E. (1934). Les espaces de finsler. Actualities 79, Paris.

Finsler, P. (1918). UberKurven and flachen in allgemeinem raumen. $\mathrm{PhD}$ Thesis, Gottingen.

Kropina, V.K. (1961). Projective two dimensional Finsler spaces with special metric. Trudy. Sem. Vektor. Tenzor. Ana, 11: 277-292. 
Matsumoto, M. \& Eguchi, K. (1974). Finsler space admitting a concurrent vector field. Tensor, N. S., 28: 239-249.

Matsumoto, M. \& Numata, S. (1980). On semi C-reducible Finsler spaces with constant coefficients and C2-like finsler spaces. Tensor, N.S., 34: $218-222$.

Matsumoto, M. \& Shimada, H. (1978). On Finsler spaces with 1-form metric . Tensor, N.S., 32 pp. 161-165.

Matsumoto, M. (1972). On C reducible Finsler spaces. Tensor, N. S., 24, pp. 29-37.

Matsumoto, M. (1986). Foundations Finsler geometry and special Finsler spaces. Kaiseisha Press Saikawa Otsu 520 Japan.

Matsumoto, M. (1992). Theory of Finsler spaces with $(\alpha, \beta)$ - metrics. Rep. Math.phy, 31: 43-83

Pandey, T.N. \& Diwedi, D.K. (1997). A theory of four dimensional Finsler Spaces in terms of Scalars. J.Nat. Acad. Math. 11, pp. 176-190.

Park, H.S. \& Lee, I.Y. (2001). The Randers changes of Finsler spaces with $(\alpha, \beta)$ - metrics of Douglas type. J. Korean Math. Soc., 38: 503-521.

Prasad, B. N. \& Kumari, B. (2013). The change of Finsler metric and imbedding classes of their tangent spaces. Tensor, N. S., 74, pp. 48-59.

Prasad, B.N. \& Singh, J.N., (1998). On C3-like finsler spaces. Ind. J. Pure, Appl. Math. 19(5): 423-428.

Prasad, B.N., Shukla, H.S., \& Joshi, O.P. (2013). The exponential change of Finsler metric and relation between imbedding class number of their tangent Riemannian spaces. Romanian Journal of Mathematicsand Computer Science. 3(1): 96-108.

Rund, H. (1959). The differential geometry of Finsler spaces. Springer Verlag.

Shibata, C. (1984). On invariant tensors of $\beta$ - change of Finsler metric. $J$. Math. Kyoto Univ., 24: 163-188. 
10 THE $\beta$-CHANGE BY FINSLER METRIC OF C-REDUCIBLE FINSLER ...

Shukla, H.S., Pandey, O.P. \& Joshi, H.D. (2012). Matsumoto change of Finsler metric. J. Int. Acad. Phys.Sci., 16(4): 329-341.

Synge, J.L. (1925). A generalization of the riemannian line element. Trans. Amer. Math. Soc., 27: 61-67.

Tachibana, S. (1950.) On Finsler spaces which admit a concurrent vector field. Tensor, N. S., 1: 1-5. 